\title{
Análise faunística de afídeos (Hemiptera, Aphididae) na Serra do Mar, Paraná, Brasil
}

\author{
Crisleide Maria Lazzarotto² \& Sonia Maria Noemberg Lazzari²
}

${ }^{1}$ Contribuição ${ }^{\circ} 1518$ do Departamento de Zoologia da Universidade Federal do Paraná.

2Departamento de Zoologia, UFPR. Caixa Postal 19020, 81531-980 Curitiba-PR, Brasil. cmlazza@ig.com.br e lazzari@ufpr.br. Bolsistas do CNPq.

\begin{abstract}
Faunistic analysis of aphids (Hemiptera, Aphididae) in the Serra do Mar, Paraná, Brazil. A total of 8,134 aphids belonging to 87 species were collected with yellow pan traps during 13 months in Serra do Mar, Paraná State. Eleven places at different altitudes $(-10 \mathrm{~m}$ to $1000 \mathrm{~m}$ above sea level - a.s.1.) were surveyed. The occurrence and dominance of the aphids were related to altitude, local flora, and environmental disruption. The number of specimens was the highest at $78 \mathrm{~m}$ and $555 \mathrm{~m}$ a.s.l., but most of the species were classified as rare. The common species registered were either polyphagous or had their preferential host plants present in the areas. The species Aphis spiraecola, Toxoptera aurantii, Brevicoryne brassicae, Tetraneura nigriabdominalis, and Uroleucon ambrosiae occurred in all surveyed areas. There was no clear relation between the faunistic data with the environmental conditions and altitude of the areas. Traps placed in transitional areas between the macroenvironments of the Dense Atlantic Montane Ombrophyllous Forest and the Mixed Ombrophyllous Forest collected the highest number of aphid species.
\end{abstract}

KEYWORDS. Aphididae; Atlantic Forest; population ecology; yellow pan traps.

RESUMO. Análise faunística de afídeos (Hemiptera, Aphididae) na Serra do Mar, Paraná, Brasil. Foram coletados na Serra do Mar, Paraná, 8134 espécimes de afídeos pertencentes a 87 espécies usando armadilhas amarelas de água durante 13 meses em onze locais com um gradiente altitudinal entre $-10 \mathrm{~m}$ sobre o nível do mar (s.n.m.) e $1000 \mathrm{~m}$ s.n.m. A ocorrência e a dominância dos afídeos foram correlacionadas com características ambientais de cada área, principalmente com a altitude, flora local e alterações antrópicas. O número mais elevado de espécimes foi entre $78 \mathrm{~m}$ e $555 \mathrm{~m}$ s.n.m., sendo a grande maioria das espécies classificada como raras. As espécies consideradas comuns coletadas em todos os locais foram as de hábito polífago e aquelas cujos hospedeiros preferenciais estavam presentes na área, como: Aphis spiraecola, Toxoptera aurantii, Brevicoryne brassicae, Tetraneura nigriabdominalis e Uroleucon ambrosiae. Não foi observada uma relação clara entre os índices faunísticos obtidos com a flora local e a altitude das áreas. As armadilhas colocadas em áreas de transição entre os macro-ambientes de Floresta Ombrófila Densa Atlântica Montana e de Floresta Ombrófila Mista capturaram o maior número de espécies de afídeos.

PALAVRAS-CHAVE. Aphididae; armadilha de Möericke; ecologia de populações; Floresta Atlântica.

Grande parte da Serra do Mar é representada por Floresta Ombrófila Densa dentro da Floresta Atlântica com características florísticas e altitudinais bastante diversificadas (Veloso \& Góes-Filho 1982). De acordo com Cox \& Moore (1993), áreas de florestas tropicais, como a Floresta Atlântica em estudo, contém uma variedade expressiva de espécies animais devido à riqueza e disponibilidade de recursos alimentares.

No Brasil, são registradas apenas 3\% das espécies de afídeos catalogadas para o mundo (Costa et al. 1993 e SousaSilva \& Ilharco 1995). Dixon et al. (1987) discutem que a pequena quantidade de afídeos nos trópicos é devida à grande quantidade de espécies de plantas, tendo os afídeos baixa eficiência em localizar seu hospedeiro. Dixon \& Kindlmann (1990) sustentam a idéia de que a abundância das plantas hospedeiras é o principal fator que determina a abundância destes insetos e, segundo Heie (1994), os afídeos são um grupo pouco especializado para explorar novos ambientes com flora diversificada. Lazzarotto \& Lazzari (1998), estudando a presença de afídeos na Serra do Mar, observaram que a maior riqueza e maior diversidade foram registradas em uma área de transição de mata primária com vegetação em diferentes estágios sucessionais a 85 m s.n.m., porém, não observaram um padrão de ocorrência em função da altitude.

Alguns trabalhos relacionam a ocorrência e dominância de algumas espécies de afídeos com o hábito polífago, presença abundante da planta hospedeira, grande atratividade pela cor amarela da armadilha e por ambientes alterados antropicamente (Blackman \& Eastop 1984; Holman 1974; Avinent et al.1991 e Webb et al.1994). Segundo Cividanes (2002), as alterações que ocorrem nas densidades populacionais de pulgões são pouco compreendidas.

O objetivo deste estudo é analisar a ocorrência de afídeos na Serra do Mar, usando os índices faunísticos de ocorrência e dominância, correlacionados com as características ambientais de cada área, principalmente com a altitude, flora local e alterações ambientais antrópicas, contribuindo, assim, para o conhecimento da afidofauna brasileira em um ecossistema natural complexo.

\section{MATERIAL E MÉTODOS}

Durante o período de dezembro de 1993 a dezembro de 1994 foram efetuadas 37 coletas com armadilhas-amarelas-de- 
água (tipo Möericke), com modificações: as armadilhas foram confeccionadas com bacias plásticas redondas de cor amarela e a borda pintada de marrom, com orifícios vedados com tela de nylon para evitar que a água transbordasse. Cada armadilha continha 2,5 L de água, com algumas gotas de detergente e 10 $\mathrm{mL}$ de formol a $10 \%$, a fim de conservar os insetos por mais tempo.

Quinzenalmente, os afídeos eram retirados das armadilhas com um pincel fino e conservados em frascos com etanol a $70 \%$ para posterior triagem e montagem. Os espécimes foram montados em lâminas permanentes, com bálsamo do Canadá, seguindo a metodologia de Martin (1983), com pequenas modificações. A identificação foi feita sob microscópio ótico, com auxílio de chaves e comparações com coleção de referência.

As coletas foram efetuadas em onze locais da Mata Atlântica nos municípios de Morretes e São José dos Pinhais, Paraná, cuja altitude e caracterização florística são descritas a seguir.

Os locais de 1 a 5 ficavam na Estação do IAPAR em Morretes, Paraná, caracterizados pela Floresta Ombrófila Densa Atlântica de Terras Baixas (Local 1, -10 m s.n.m.; Local 2, 10 m s.n.m.; Local 3, 20 m s.n.m.) e Floresta Ombrófila Densa Atlântica Submontana (Local 4, 65 m s.n.m.; Local 5, 78 m s.n.m.). Nos locais 1 e 2 as armadilhas foram instaladas em áreas abertas sendo influenciadas por vegetação secundária bastante avançada. Nos locais 3, 4 e 5, as armadilhas foram instaladas em meio à vegetação secundária em estágios inicial e médio de desenvolvimento.

Os locais 6, 8, 9 e 10 ficavam na Estrada da Graciosa, no município de Morretes, PR, sendo o 6 (85 m s.n.m.), 8 (190 m s.n.m.) e 9 (555 m s.n.m.) caracterizados como Floresta Ombrófila Densa Atlântica Submontana, com influência de vegetação secundária e predomínio de plantas herbáceas; o local 10 (750 m s.n.m.) caracterizava-se pela transição entre Floresta Ombrófila Mista (Floresta de Araucária) e Floresta Ombrófila Densa Atlântica (Floresta Atlântica) adjacente a uma área com vegetação de estágios sucessionais iniciais (espécies pioneiras).

Na BR277 (Rodovia Curitiba - Paranaguá) foram estabelecidos o Local 7 (185 m s.n.m.), no município de Morretes e o 11 (1000 m s.n.m.), em São José dos Pinhais. Essas áreas caracterizam-se como Floresta Ombrófila Densa Atlântica Montana em transição com a Floresta Ombrófila Mista. No Local 7 havia espécies frutíferas cultivadas e uma ampla área de pastagem; o 11 era circundado por áreas de Floresta Secundária.

Os macro-ambientes considerados nesta pesquisa foram agrupados pela seguinte classificação: Floresta Ombrófila Densa de Terras Baixas, representado pelos locais 1, 2 e 3 (A); Floresta Ombrófila Densa Atlântica Submontana, representado pelos locais 4, 5, 6, 8, e 9 (B); Floresta Ombrófila Densa Atlântica em transição com a Floresta Ombrófila Mista (Floresta de Araucária), representado pelo Local 10 (C) e Floresta Ombrófila Densa Atlântica Montana em transição com a Floresta Ombrófila Mista, representado pelos locais 7 e 11 (D).
Para a análise faunística foram utilizados os índices de ocorrência e dominância dos afídeos coletados com as armadilhas-amarelas-de-água, baseando-se no método proposto por Palma (1975) apud Abreu \& Nogueira (1989). A ocorrência foi obtida através da divisão entre o número de coletas onde foi registrada a espécie e o número total de coletas realizadas multiplicado por 100 . Classificou-se o número obtido em espécie acidental (0-25\%), espécie acessória (26-50\%) e espécie constante (51-100\%). A dominância foi obtida pela divisão entre o número de indivíduos da espécie e o número total de indivíduos coletados multiplicado por 100. O total obtido era classificado em espécie acidental $(0,0-2,5 \%)$, espécie acessória $(2,6-5,0 \%)$ e espécie dominante $(5,1-100 \%)$. Combinando-se os índices de ocorrência e dominância, obtevese a seguinte classificação geral ou status das espécies: espécie comum (constante + dominante), espécie intermediária (acidental + dominante; acidental + acessória; acessória + acessória ou acessória + dominante) e espécie rara (acidental + acidental).

\section{RESULTADOS E DISCUSSÃO}

Foi coletado o total de 8134 espécimes de afídeos pertencentes a 87 espécies nas armadilhas amarelas durante os 13 meses de coleta na Serra do Mar, representando 60\% das espécies de afídeos registradas para o Brasil. Mello (1994), utilizando a mesma metodologia de coleta, registrou o total de 32348 exemplares de 71 espécies, em área de cultivo de olerícolas na altitude de $930 \mathrm{~m}$ s.n.m., no município de Piraquara, PR, no mesmo período de coleta. A quantidade de afídeos coletada naquele experimento é sem dúvida bastante superior ao registrado neste estudo, porém, o número de espécies foi maior na Serra do Mar. Segundo Dixon \& Kindlmann (1990), há uma maior quantidade de pulgões em regiões onde a riqueza de plantas hospedeiras é menor, como acontece em monoculturas, pois, a eficiência dos afídeos em encontrar suas plantas hospedeiras é mais baixa onde há maior diversidade e menor densidade das espécies vegetais.

Do total de afídeos coletados, 8019 (98,6\%) exemplares eram alados, 38 ápteros, 24 ninfas e 53 machos. Para o cálculo dos índices faunísticos foram utilizados somente os exemplares alados, uma vez que a eficiência da armadilha está voltada para a afidofauna aérea local.

Observa-se na Tabela I que as espécies raras representaram $73 \%$ das ocorrências, as intermediárias $18 \%$ e as comuns $9 \%$.

As espécies de afídeos capazes de explorar e de colonizar diversas famílias de plantas são consideradas como tendo hábito polífago. Assim, nove espécies polífagas, segundo Blackman \& Eastop (1984), foram coletadas, dentre as quais, apenas $A$. spiraecola e T. aurantii estiveram presentes em todos os locais (Tabela I). Não somente o hábito polífago de A. spiraecola, mas também a sua alta atratividade pela cor amarela da armadilha (Avinent et al., $1991 \mathrm{e} \mathrm{Webb} \mathrm{et} \mathrm{al.,} \mathrm{1994),}$ provavelmente, levaram-na a ser a espécie mais representativa com o status de espécie comum na maioria dos locais.

A espécie T. aurantii foi comum em seis dos locais 
Tabela I. Lista das espécies capturadas com armadilhas-amarelas-de-água com suas respectivas classificações gerais de Palma, referentes a 11 pontos de coleta na Serra do Mar, PR, no período de dezembro de 1993 a dezembro de 1994 (Locais 01 a 09 e 11) e de abril de 1994 a dezembro de 1994 (Local 10). C = Comum; I = Intermediária; $\mathrm{R}=$ Rara.

\begin{tabular}{|c|c|c|c|c|c|c|c|c|c|c|c|}
\hline Espécies & 01 & & & & 5 & 0 & 07 & 08 & & 101 & \\
\hline Acyrthosiphon bidenticola & $\mathrm{R}$ & - & $\mathrm{R}$ & $\mathrm{R}$ & $\mathrm{R}$ & I & $\mathrm{R}$ & $\mathrm{R}$ & $\mathrm{R}$ & & $\mathrm{R}$ \\
\hline Acyrthosiphon kondoi & & - & $\mathrm{R}$ & & - & - & - & - & & _ & 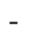 \\
\hline Acyrthosiphon malvae & - & - & - & $\mathrm{R}$ & - & - & - & - & $\mathrm{R}$ & - & - \\
\hline Acyrthosiphon pisum & $\mathrm{R}$ & - & 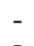 & 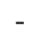 & $\mathrm{R}$ & $\mathrm{R}$ & - & - & - & & - \\
\hline phis coreopsidis & - & $\mathrm{R}$ & I & $\mathrm{R}$ & $\mathrm{C}$ & $\mathrm{R}$ & $\mathrm{R}$ & $\mathrm{R}$ & - & $-\mathrm{r}$ & $\mathrm{R}$ \\
\hline *Aphis fabae & - & $\mathrm{R}$ & - & - & $\mathrm{R}$ & - & - & - & _ & $\mathrm{R} \mathrm{R}$ & $\mathrm{R}$ \\
\hline Aphis fabae (g1 & - & - & - & - & $\mathrm{R}$ & - & - & - & - & . & - \\
\hline phis forbesi & - & $\mathrm{R}$ & - & - & - & - & - & - & $\mathrm{R}$ & $-\mathrm{r}$ & $\mathrm{R}$ \\
\hline *Aphis & I & $\mathrm{C}$ & I & I & $\mathrm{C}$ & $\mathrm{I}$ & $\mathrm{C}$ & I & $\mathrm{I}$ & $\mathrm{I}$ & 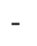 \\
\hline Aphis nerii & $\mathrm{R}$ & $\mathrm{R}$ & $\mathrm{R}$ & I & I & $\mathrm{R}$ & $\mathrm{R}$ & - & - & - & 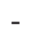 \\
\hline Aphis & - & - & 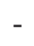 & - & $\mathrm{R}$ & - & - & $\mathrm{R}$ & $\mathrm{R}$ & - & . \\
\hline Aphis & $\mathrm{R}$ & $\mathrm{R}$ & $\mathrm{R}$ & - & $\mathrm{R}$ & $\mathrm{R}$ & - & $\mathrm{R}$ & - & - & . \\
\hline *Aphis solane & $\mathrm{R}$ & $\mathrm{R}$ & $\mathrm{R}$ & I & $\mathrm{C}$ & $\mathrm{R}$ & $\mathrm{R}$ & - & - & & $\mathrm{R}$ \\
\hline *Aphis spiraecola & $\mathrm{C}$ & $\mathrm{C}$ & $\mathrm{C}$ & $\mathrm{C}$ & $\mathrm{C}$ & $\mathrm{C}$ & $\mathrm{C}$ & $\mathrm{C}$ & $\mathrm{C}$ & $\mathrm{C} \mathrm{I}$ & I \\
\hline Aphis terricola & - & - & $\mathrm{R}$ & - & - & - & - & - & - & - & - \\
\hline Aphis & - & $\mathrm{R}$ & $\mathrm{R}$ & $\mathrm{R}$ & $\mathrm{R}$ & $\mathrm{R}$ & - & - & $\mathrm{R}$ & R R & $\mathrm{R}$ \\
\hline *Aulace & - & $\mathrm{R}$ & $\mathrm{R}$ & - & $\mathrm{R}$ & $\mathrm{R}$ & $\mathrm{R}$ & I & $\mathrm{R}$ & R $\mathrm{F}$ & $\mathrm{R}$ \\
\hline Brachyc & $\mathrm{R}$ & I & $\mathrm{R}$ & $\mathrm{R}$ & $\mathrm{C}$ & $\mathrm{R}$ & - & $\mathrm{R}$ & $\mathrm{I}$ & R I & I \\
\hline Brachycaudus & - & - & - & $\mathrm{R}$ & - & - & - & - & - & - & 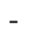 \\
\hline $\mathrm{Brac}$ & $\mathrm{R}$ & - & 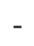 & - & - & $\mathrm{R}$ & - & - & - & - & - \\
\hline$B r e$ & $\mathrm{R}$ & $\mathrm{R}$ & I & I & $\mathrm{R}$ & I & $\mathrm{R}$ & $\mathrm{R}$ & $\mathrm{I}$ & I I & I \\
\hline Capi & - & $\mathrm{R}$ & - & - & $\mathrm{R}$ & - & - & - & - & - & . \\
\hline Capitophor & $\mathrm{R}$ & $\mathrm{R}$ & $\mathrm{R}$ & - & $\mathrm{R}$ & $\mathrm{R}$ & $\mathrm{R}$ & $\mathrm{R}$ & $\mathrm{R}$ & - & - \\
\hline Carolinaia & - & - & - & - & - & - & - & & - & $-\mathrm{r}$ & $\mathrm{R}$ \\
\hline Cave & - & - & - & - & $\mathrm{R}$ & $\mathrm{R}$ & $\mathrm{R}$ & - & $\mathrm{R}$ & - & 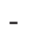 \\
\hline Cera & - & - & 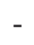 & $\mathrm{R}$ & - & - & $\mathrm{R}$ & & - & - & - \\
\hline aritimae & - & - & - & - & - & $\mathrm{R}$ & - & & $\mathrm{R}$ & - & - \\
\hline Colo & $\mathrm{R}$ & - & - & - & - & $\mathrm{R}$ & - & & $\mathrm{R}$ & R R & $\mathrm{R}$ \\
\hline Dysa & - & - & 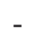 & $\mathrm{R}$ & - & $\mathrm{R}$ & - & - & - & - & - \\
\hline Dysaphis foeniculus & - & $\mathrm{R}$ & - & - & - & $\mathrm{R}$ & - & & - & - & . \\
\hline Eulachnus rileyi & - & $\mathrm{R}$ & - & $\mathrm{R}$ & - & $\mathrm{R}$ & $\mathrm{R}$ & & $\mathrm{R}$ & $-\mathrm{r}$ & $\mathrm{R}$ \\
\hline Geoica lucifuga & - & - & 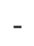 & - & - & - & - & - & - & $-\mathrm{r}$ & $\mathrm{R}$ \\
\hline phigus floccosus & $\mathrm{R}$ & $\mathrm{R}$ & $\mathrm{R}$ & - & $\mathrm{R}$ & $\mathrm{I}$ & $\mathrm{R}$ & $\mathrm{R}$ & $\mathrm{I}$ & - & - \\
\hline Hyadaphis foeniculi & - & $\mathrm{R}$ & 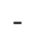 & - & $\mathrm{R}$ & - & - & - & - & - & 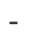 \\
\hline Hyper & - & - & - & - & - & - & - & & - & $-\mathrm{r}$ & $\mathrm{R}$ \\
\hline Hyperomyzus lactucae & $\mathrm{R}$ & - & . & $\mathrm{R}$ & $\mathrm{R}$ & $\mathrm{R}$ & - & & $\mathrm{R}$ & $-\mathrm{r}$ & 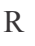 \\
\hline Lipaphis erysimi & $\mathrm{R}$ & I & $\mathrm{R}$ & I & I & $\mathrm{I}$ & $\mathrm{R}$ & $\mathrm{R}$ & I & - & D \\
\hline Lizerius cermelii & - & - & - & - & - & - & $\mathrm{R}$ & - & - & - & - \\
\hline Lizerius intermedius & - & - & - & - & - & $\mathrm{R}$ & - & - & - & - & 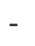 \\
\hline Lizerius tuberculatus & - & $\mathrm{R}$ & - & - & - & $\mathrm{R}$ & $\mathrm{R}$ & - & - & $\mathrm{R}$ & - \\
\hline Macrosiphini sp. 1 & - & - & - & - & - & - & - & - & $\mathrm{R}$ & - & - \\
\hline
\end{tabular}

\begin{tabular}{|c|c|c|c|c|c|c|c|c|c|c|}
\hline Espécies & 01 & & & & & & & 0809 & & 011 \\
\hline Macrosiphini sp. 2 & - & - & - & - & - & - & - & - & - & $\mathrm{R}$ \\
\hline Macrosiphini sp. 3 & - & - & - & $\mathrm{R}$ & - & - & - & $-\quad-$ & $-\quad-$ & - \\
\hline Macrosiphoniella yomogifoliae & - & - & - & - & $\mathrm{R}$ & - & - & $-\quad-$ & - - & $\mathrm{R}$ \\
\hline *Macrosiphum euphorbiae & $\mathrm{R}$ & - & - & - & - & - & $\mathrm{R}$ & - & - & 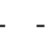 \\
\hline Melanaphis sacchari & - & - & - & - & - & - & _ & & $\mathrm{R}-$ & - \\
\hline Metopolophium dirhodum & - & - & - & - & $\mathrm{R}$ & - & - & $-\quad-$ & $-\quad-$ & $-\quad-$ \\
\hline Microparsus brasiliensis & - & - & - & - & - & $\mathrm{R}$ & $\mathrm{R}$ & $-\quad-$ & $-\quad-$ & - \\
\hline Microparsus vignaphillus & $\mathrm{R}$ & - & - & - & - & $\mathrm{R}$ & - & & $\mathrm{R}-$ & $-R$ \\
\hline Myzocallis castanicola & - & - & - & - & - & $\mathrm{R}$ & - & $-\quad-$ & $-\quad-$ & - $\mathrm{R}$ \\
\hline Myzus hemerocallis & - & - & - & - & - & - & $\mathrm{R}$ & & $\mathrm{R}-$ & - \\
\hline *Myzus ornatus & - & $\mathrm{R}$ & - & - & $\mathrm{R}$ & $\mathrm{R}$ & $\mathrm{R}$ & & $\mathrm{R}-$ & $\mathrm{R}$ \\
\hline *Myzus persicae & I & $\mathrm{R}$ & $\mathrm{R}$ & - & $\mathrm{C}$ & I & $\mathrm{C}$ & $\mathrm{I} R$ & $\mathrm{R}-$ & $-\quad I$ \\
\hline Ovatus crataegarius & - & - & - & - & - & $\mathrm{R}$ & - & $-\quad-$ & - - & - \\
\hline Pemphigus populitransversus & $\mathrm{R}$ & - & - & $\mathrm{R}$ & $\mathrm{R}$ & $\mathrm{R}$ & $\mathrm{R}$ & & $\mathrm{R}-$ & $-\mathrm{R}$ \\
\hline Pentalonia nigronervosa & $\mathrm{R}$ & $\mathrm{R}$ & I & $\mathrm{R}$ & $\mathrm{R}$ & I & $\mathrm{C}$ & $\mathrm{I} R$ & $\mathrm{R}-$ & $-\quad-$ \\
\hline Rhopalosiphina & $\mathrm{R}$ & - & - & - & - & $\mathrm{R}$ & $\mathrm{R}$ & $-\quad-$ & - - & - \\
\hline Rhopalosiphoninus latysiphon & - & - & - & - & $\mathrm{R}$ & - & $\mathrm{R}$ & & $-\quad-$ & $-R$ \\
\hline Rhopalosiphum maidis & $\mathrm{R}$ & I & $\mathrm{R}$ & $\mathrm{R}$ & I & $\mathrm{R}$ & $\mathrm{I}$ & I I & I - & - $\mathrm{R}$ \\
\hline Rhopalosiphum nymphaeae & - & $\mathrm{R}$ & - & - & $\mathrm{R}$ & - & - & & - & - $\mathrm{R}$ \\
\hline Rhopalosiphum padi & $\mathrm{R}$ & $\mathrm{R}$ & $\mathrm{R}$ & $\mathrm{R}$ & $\mathrm{R}$ & $\mathrm{R}$ & I & & $-R$ & $\mathrm{R}$ \\
\hline Rhopalosiphum & & & & & & & & & & \\
\hline rufiabdominale & $\mathrm{R}$ & $\mathrm{R}$ & $\mathrm{R}$ & $\mathrm{R}$ & I & $\mathrm{R}$ & $\mathrm{R}$ & $\mathrm{R} \mathrm{R}$ & $\mathrm{R}-$ & $\mathrm{R}$ \\
\hline Schizaphis gram & $\mathrm{R}$ & $\mathrm{R}$ & $\mathrm{R}$ & - & - & $\mathrm{R}$ & $\mathrm{R}$ & & $\mathrm{R}-$ & $-R$ \\
\hline Sipha flava & $\mathrm{R}$ & $\mathrm{R}$ & - & - & - & $\mathrm{R}$ & - & & $-\mathrm{R}$ & $\mathrm{R}$ \\
\hline Sitobion avenae & - & - & - & - & $\mathrm{R}$ & $\mathrm{R}$ & - & & $\mathrm{R}-$ & $\mathrm{R}$ \\
\hline Sitobion lambersi & - & - & - & $\mathrm{R}$ & - & - & - & & $-\quad-$ & $-R$ \\
\hline Sitobion luteum & - & - & - & - & - & $\mathrm{R}$ & - & - - & - - & - $\mathrm{R}$ \\
\hline Sitobion salviae & $\mathrm{R}$ & - & - & - & - & - & - & $-\quad-$ & $-\quad-$ & $-\quad-$ \\
\hline Tetraneura nigriabdominalis & I & I & $\mathrm{R}$ & $\mathrm{R}$ & $\mathrm{R}$ & $\mathrm{C}$ & I & I I & I I & I $\mathrm{C}$ \\
\hline Therioaphis trifolii & - & - & - & - & - & - & - & $-\quad-$ & $-\quad-$ & $-R$ \\
\hline *Toxoptera aurantii & $\mathrm{C}$ & $\mathrm{C}$ & $\mathrm{C}$ & $\mathrm{C}$ & $\mathrm{R}$ & $\mathrm{C}$ & $\mathrm{C}$ & I I & I C & $\mathrm{C} \mathrm{R}$ \\
\hline Toxoptera citricidus & $\mathrm{C}$ & $\mathrm{C}$ & I & $\mathrm{R}$ & - & I & $\mathrm{C}$ & $\mathrm{I} C$ & $\mathrm{C} \mathrm{R}$ & R I \\
\hline Tuberolachnus salignus & - & - & - & - & - & $\mathrm{R}$ & - & $-\quad-$ & $-\mathrm{R}$ & R - \\
\hline Uroleucon ambrosiae & I & I & I & I & $\mathrm{R}$ & I & I & I I & $\mathrm{I} R$ & $\mathrm{R}$ \\
\hline Uroleucon compositae & - & $\mathrm{R}$ & $\mathrm{R}$ & - & $\mathrm{R}$ & - & $\mathrm{R}$ & & $-\quad-$ & $-\quad-$ \\
\hline Uroleucon erigeronensis & - & - & - & - & $\mathrm{R}$ & - & - & & $\mathrm{R}-$ & $-\quad-$ \\
\hline Uroleucon gravicorne & - & - & - & - & - & - & - & & $-\quad-$ & $-\mathrm{R}$ \\
\hline Uroleucon mиеттоsит & - & - & - & - & - & - & - & & $-R$ & R - \\
\hline Uroleucon sonchi & - & - & - & - & - & $\mathrm{R}$ & $\mathrm{R}$ & $-\mathrm{R}$ & $\mathrm{R}-$ & $-\mathrm{R}$ \\
\hline Uroleucon sp. 1 & - & - & - & - & - & - & - & $\mathrm{R}-$ & $-\quad-$ & $-\quad-$ \\
\hline
\end{tabular}

* Espécies polífagas segundo Blackman \& Eastop 1984. amostrados, intermediária nos locais 08 e 09 e rara nos locais 05 e 11 . No estudo de Avinent et al. (1991), que relacionam, comparativamente, armadilhas de cor verde e amarela, o maior número de T. aurantii foi registrado nas armadilhas verdes, mas sem diferenças estatisticamente significativas entre as duas colorações para a referida espécie. Já, Seif(1988) obteve, em seu estudo dos afídeos de citros no Kenya, que T. aurantii responde mais às armadilhas amarelas do que às verdes.

O pulgão-do-algodoeiro, A. gossypii, é considerada uma espécie bastante comum e polífaga (Blackman \& Eastop 1984), no entanto, obteve o status de espécie comum em apenas três dos locais amostrados (02, 05 e 07), os quais possuem um nível de alteração antrópica maior do que os outros. Estes resultados corroboram com Holman (1974), que registrou esta espécie mais comumente em áreas alteradas pela atividade humana. Foi ausente na altitude mais elevada, concordando também com esse autor, que constatou sua raridade em zonas mais altas. Avinent et al. (1991) e Halbert et al. (1986) constataram que a porcentagem de A. gossypii foi significativamente maior em armadilhas verdes do que nas 
amarelas; já Webb et al. (1994) não encontraram diferença significativa na preferência de armadilhas amarelas ou verdes para a referida espécie.

As demais espécies polífagas, Aphis fabae, Aphis solanella, Aulacorthum solani, Macrosiphum euphorbiae, Myzus ornatus e M. persicae, não estiveram presentes em todos os locais, sendo consideradas raras na maioria dessas áreas (Tabela I).

Mello (1994) registrou seis espécies comuns de acordo com a classificação de Palma, em armadilhas amarelas, em área de olerícolas no Paraná: A. gossypii, A. spiraecola, $B$. brassicae, L. erysimi, M. persicae, T. citricidus e U. ambrosiae. Nesta pesquisa, A. spiraecola obteve o status comum para quase todos os locais, exceto para o Local 11, onde se classificou como intermediária; enquanto que T. citricidus foi comum em até quatro locais e as demais espécies, não foram comuns em nenhum dos locais amostrados. Carvalho et al. (2002) encontraram cinco espécies constantes (presentes em mais de $50 \%$ das coletas) de acordo com a classificação de Bodenheimer, em cultivo de hortaliças em Lavras, MG: Lipaphis erysimi, Toxoptera citricidus e Aphis spp. (A. fabae, A. gossypii e A. spiraecola).

As espécies não polífagas de acordo com Blackman \& Eastop (1984) que se alimentam de uma família de plantas, e que ocorreram em todos os locais, foram: B. brassicae, $T$. nigriabdominalis e $U$. ambrosiae, tendo como hospedeiros preferenciais as plantas das famílias Brassicaceae, Poaceae e Asteraceae, respectivamente. A presença dessas plantas hospedeiras em todos os locais de coleta proporcionou a presença dos afídeos. A espécie $B$. brassicae foi intermediária em locais onde o calor não era tão intenso, mas foi rara nos locais mais quentes e abafados, concordando com Holman (1974), que observou que nos trópicos esta espécie é mais comum nas montanhas e áreas com clima moderado.

A espécie T. nigriabdominalis, que coloniza raízes de gramíneas, foi comum nos locais com o predomínio de Poaceae próximo às armadilhas; sendo classificada como rara onde havia, além de gramíneas, diversas plantas de outras famílias, o que poderia dificultar ao pulgão a localização de sua planta hospedeira (Dixon \& Kindlmann 1990).

De acordo com o agrupamento resultante da caracterização dos locais de coleta, observa-se que dos quatro macroambientes estudados, os ambientes C e D (Floresta Ombrófila Densa Atlântica e Montana com Floresta Ombrófila Mista) destacaram-se em relação à média do número de espécies (Tabela II). Segundo Odum (1988), uma transição nítida entre duas ou mais comunidades vegetais é denominada de ecótone e freqüentemente há uma tendência de o número de espécies animais e a densidade populacional de algumas destas espécies serem maiores; sendo esta tendência conhecida como efeito de borda. Em relação ao número de espécimes de afídeos, a média encontrada não apresentou uma diferença relevante, uma vez que o ambiente $\mathrm{C}$, apesar de apresentar o menor número de afídeos em relação aos outros ambientes, teve uma defasagem de quatro meses de coletas e foi representado apenas pelo Local 10 .
Tabela II. Agrupamento dos 11 locais de coletas de afídeos em macroambientes na Serra do Mar, PR, no período de dezembro de 1993 a dezembro de 1994 (Locais 01 a 09 e 11) e de abril de 1994 a dezembro de 1994 (Local 10).

\begin{tabular}{cccc}
\hline $\begin{array}{c}\text { Macro- } \\
\text { ambiente }\end{array}$ & $\begin{array}{c}\text { No Médio de } \\
\text { espécimes }\end{array}$ & $\begin{array}{c}\text { No Médio de }^{\circ} \\
\text { espécies }\end{array}$ & $\begin{array}{c}\text { Espécies } \\
(\%)\end{array}$ \\
\hline A & 713 & 15 & 20 \\
B & 841 & 14 & 18.9 \\
C & 256 & 18 & 24 \\
D & 709 & 27 & 36 \\
\hline
\end{tabular}

A - Floresta Ombrófila Densa Atlântica de Terras Baixas - Locais 1, 2 e 3; B - Floresta Ombrófila Densa Atlântica Submontana - Locais 4, 5, 6, 8 e 9; C - Floresta Ombrófila Densa Atlântica x Floresta Ombrófila Mista - Local 10; D - Floresta Ombrófila Densa Atlântica Montana x Floresta Ombrófila Mista - Locais 7 e 11.

Pelos resultados obtidos conclui-se que o maior número das espécies de afídeos ocorre na área de transição da Floresta Ombrófila Densa Atlântica Montana com a Floresta Ombrófila Mista. Espécies consideradas polífagas, como A. spiraecola e T. aurantii estão presentes em todos os locais de coleta em função da diversidade de hospedeiros que exploram. Da mesma forma, $B$. brassicae, T. nigriabdominalis e $U$. ambrosiae, que são monófagas ou oligófagas, são abundantes devido à presença de suas plantas hospedeiras preferenciais nas áreas.

Uma análise complementar da relação afídeo-planta hospedeira e dos fatores climáticos são importantes para estabelecer um padrão de ocorrência e distribuição dos afídeos no ambiente.

\section{REFERÊNCIAS}

Abreu, P. C. O. V. \& C. R. Nogueira. 1989. Spatial distribution of species at Rio de Janeiro Coast, Brasil. Ciência e Cultura 41 897-902.

Avinent, L.; A. Hermoso de Mendoza \& G. Llácer. 1991. Comparison of traps for capture of alate aphids (Homoptera, Aphidinea) in apricot tree orchards. Agronomie 11: 613-618.

Blackman, R. L. \& V. F. Eastop. 1984. Aphids on the World's Crops - An identification guide. Wiley \& Sons, 466p.

Carvalho, L. M.; V. H. P. Bueno \& R. P. Martinez. 2002. Levantamento de afídeos alados em plantas hortícolas em Lavras - MG. Ciência Agrotecnológica 26: 523-532.

Cividanes, F. J. 2002. Impacto de inimigos naturais e de fatores meteorológicos sobre uma população de Brevicoryne brassicae (L.) (Hemiptera: Aphididae) em couve. Neotropical Entomology 31: 249-255.

Costa, C. L.; V. F. Eastop \& R. L. Blackman. 1993. Brazilian Aphidoidea: I. Key to families, subfamilies and account of the Phylloxeridae. Pesquisa Agropecuária Brasileira 28: 197-215.

Cox, C. B. \& P. D. Moore. 1993. Biogeography - An ecological and evolutionary approach. $5^{\text {a }}$ ed. Blackwell Science Ltd. London.

Dixon, A. F. G.; P. Kindlmann; J. Leps \& J. Holman. 1987. Why there are so few species of aphids, especially in the tropics. The American Naturalist 129: 580-592.

Dixon, A. F. G. \& P. Kindlmann. 1990. Role of plant abundance in determining the abundance of herbivorous insects. Oecologia 83: $281-283$. 
Halbert, S. E.; G-X. Zhang \& Z-Q. Pu. 1986. Comparison of sampling methods for alate aphids and observations on epidemiology of soybean mosaic virus in Nanjing, China. Annals Applied Biology 109: 473-483.

Heie. O. E. 1994. Why are there so few aphid species in the temperate areas of the southern hemisphere? European Journal Entomology 91: 127-133.

Holman, J. 1974. Los áfidos de Cuba. Instituto Cubano del Libro, La Habana, 304p.

Lazzarotto, C. M. \& Lázzari, S. M. N. 1998. Richness and diversity of aphids (Homoptera, Aphididae) along an altitudinal gradient in the Serra do Mar, Paraná, Brazil. Revista Brasileira de Zoologia 15: $977-983$.

Martin, J. H. 1983. The identification of common aphid pests of tropical agriculture. Tropical Pest Management 29: 395-411.

Mello, M. E. F. 1994. Afídeos (Homoptera: Aphididae) e seus inimigos naturais em olerícolas, Piraquara, Paraná. Tese de Mestrado.
Universidade Federal do Paraná. Curitiba, 85p.

Odum, E. P. 1988. Ecologia. Ed. Guanabara. Rio de Janeiro, 434p.

Seif, A. A. 1988. Comparison of green and yellow water traps for sampling citrus aphids at the Kenya Coast. East African Agricultural and Forestry Journal 53: 159-161.

Sousa-Silva, C. R. \& F. A. Ilharco. 1995. Afídeos do Brasil e suas plantas hospedeiras (lista preliminar). São Carlos: EDUFSCar, $85 \mathrm{p}$.

Veloso, H. P. \& L. Góes-Filho. 1982. Fitogeografia Brasileira, Classificação Fisionômica-Ecológica da Vegetação Neotropical. Boletim Técnico. Projeto Radambrasil. Série Vegetação. Salvador, 79p.

Webb, S. E.; M. L. Kok-Yokomi \& D. J. Voegtlin. 1994. Effect of trap color on species composition of alate aphids (Homoptera: Aphididae) caught over watermelon plants. Florida Entomologist 77: $146-154$. 